\title{
Vortex-Induced Vibration Tests of a Marine Growth Wrapped Cylinder at Subcritical Reynolds Number
}

\author{
V. J. Kurian ${ }^{1, *}, A . M$. Al-Yacouby ${ }^{1}$, and A. R. M. Khir ${ }^{1}$ \\ ${ }^{I}$ Department of Civil and Environmental Engineering, Universiti Teknologi PETRONAS, 32610 \\ Bandar Seri Iskandar, Perak, Malaysia
}

\begin{abstract}
Vortex Induced Vibrations (VIV) may cause great damage to deep water risers. Estimation of accurate hydrodynamic coefficients and response amplitudes for fouled tubular cylinders subjected to VIVs is a complex task. This paper presents the results of an extensive experimental investigation on in-line and cross-flow forces acting on cylinders wrapped with marine growth, subjected to current at Subcritical Reynolds Number. The drag and lift force coefficients have been determined through the use of the Fast Fourier Analysis methods. The different tests were conducted in the offshore engineering laboratory at Universiti Teknologi PETRONAS (UTP), Malaysia. In this study, a cylinder with outer diameter $D o=27 \mathrm{~mm}$, fixed at top as cantilever beam was used. The in-line and cross-flow forces were measured using VIV Force Totaller (VIVFT). VIVFT is a two degree of freedom (2DOF) forces sensor developed by UTP to measure the VIV forces. The tests were conducted for current velocity varied between 0.118 to $0.59 \mathrm{~m} / \mathrm{s}$. The test results suggest that the cylinder wrapped with marine growth has shown an overall increase in drag and inertia coefficients as well as on response amplitudes.
\end{abstract}

\section{Introduction}

Accurate estimation of VIVs of rough circular cylinder is one of the most difficult task in offshore engineering, due to the complex and non-linear interaction of rough cylinder with the surrounding fluid. VIV of slender cylinder is of practical importance to many fields of engineering. The forces acting on the cylinders experience periodic change due to the vortex shedding. Vibrations resulting from the vortex shedding in the wake of a cylinder exposed to flow can create fatigue damage in production risers used by the offshore oil and gas industry. VIV phenomenon is a function of various parameters, the two main parameters are the Reynolds number and the Strouhal Number, St. The Reynolds number is defined as $R e=U D / v$ where $v=10^{-6} \mathrm{~m}^{2} / \mathrm{s}$ is the kinematic viscosity of water at room

\footnotetext{
* Corresponding author: kurian_john@petronas.com.my
} 
temperature and the Strouhal number is defined as $S t=f_{s} D / U$, where $U$ is the fluid velocity, $D$ is the diameter of the cylinder and $f_{s}$ is the shedding frequency. The fluid-structure coupling is characterized by the reduced velocity $U_{r}=U / f_{s} D$. For instance Feng [1-6] investigated the VIV of circular cylinders. The pioneering experimental investigation conducted to assess the effects of surface roughness on the pressure distribution around a bluf body was conducted by Fage and Warsap [7].

Further, Hover et al. [8] conducted experimental study to investigate the effects of thin wires attached on the outer surface and parallel to the axis of a smooth circular cylinder in a steady flow. The reader can also refer to [9-10] for further study on VIVs. However, the effects of marine growth and surface roughness on VIV considering the Malaysian water is not existing. Therefore, the objective of this paper is to assess the effect of marine growth on VIV forces, response amplitudes and the most important flow and structural characteristics governing VIVs, taking into account the marine growth characteristics for the Malaysia offshore locations. According to PTS [11] the marine growth layer thickness to be considered varies from 25 to $127 \mathrm{~mm}$, while the corresponding surface roughness varies from 6.25 to $64 \mathrm{~mm}$, depending on the geographical location and the water depth. Dry unit weight of marine growth recommended for the Malaysian water is $10 \mathrm{kN} / \mathrm{m}^{3}$ [11].

\section{Research methodology}

\subsection{Details of the wave tank}

The VIV model test was conducted in the wave basin of Universiti Teknologi PETRONAS. The wave basin where the tests were carried out is $20 \mathrm{~m}$ length $\times 10 \mathrm{~m}$ wide $\times 1 \mathrm{~m}$ water depth $(d)$. The wave tank is equipped with 16 wave paddles capable of generating regular, random and multidirectional waves. Besides, the wave tank is equipped with pumps capable of generating currents. However, as the maximum velocity of current in the wave tank was limited to $0.12 \mathrm{~m} / \mathrm{s}$ at a maximum water depth of $1 \mathrm{~m}$, a test section was constructed inside the wave tank to increase the current velocity to meet the subcritical flow regimes requirements. Fig. 1a shows a close view of the test section, while Fig. 1b depicts a sectional view of the test section. The response amplitudes of the cylinder due to VIV were measured using OptiTrack motion sensing system, while the current velocity was measured using VECTRINO velocity meter. Due to the limitations of testing facility, a scale factor of 1:55 was adopted and the test results are presented in terms of prototype for engineering applications.

The variation of marine growth thickness with water depth was varied as $3: 2: 1$. The profile of marine growth adopted in this study represents the general pattern of marine growth accumulation on circular cylinders adopted for Malaysian water applications. The surface roughness of marine growth was achieved by wrapping the wet length of the cylinder by sand paper with surface roughness, $k=1 \mathrm{~mm}$. The model is connected to a two degree of freedom (2DOF) VIV force sensor specially designed and developed to capture the total VIV forces. The recorded forces have been analysed and the corresponding drag and lift coefficients have been determined using the well-known Morison equation components as described in Section 3.1.

Details of model set up in the wave tank and the VIV force sensor are presented in Fig 2. The VIV experiments were carried out in a test section of $3 \mathrm{~m}$ long $\times 1.5 \mathrm{~m}$. Totally 270 different tests were conducted in the wave tanks. The environmental conditions generated in the wave tank corresponding to the deferent test conditions denoted as T1 to T5 are presented in Table 1. 

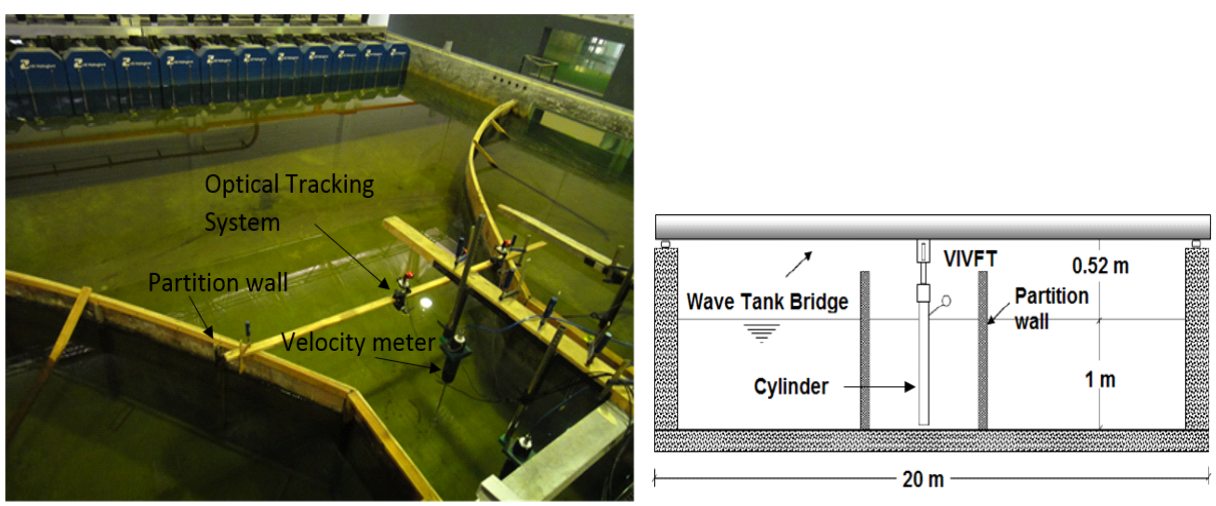

Fig. 1. a) A close view of the wave basin and b) a sectional view of the test section
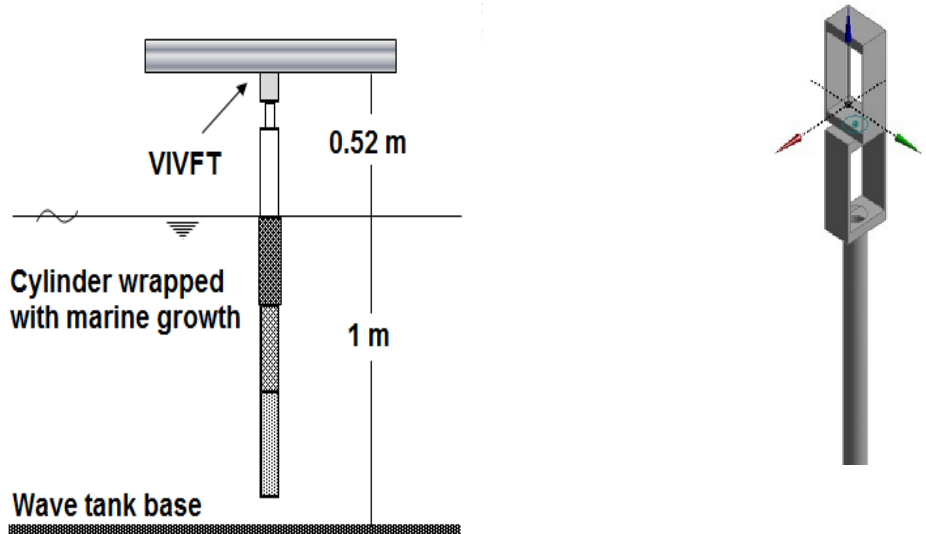

a) Model set up in the wave tank

b) Perspective view of a cylinder fitted with VIV force sensor

Fig. 2. Details of model set up and VIV force sensor

Table 1. Variation of current velocity generated in the wave tank

\begin{tabular}{|c|c|}
\hline Test Condition & Current Velocity $(\mathbf{m} / \mathbf{s})$ \\
\hline T1 & 0.12 \\
\hline T2 & 0.22 \\
\hline T3 & 0.32 \\
\hline T3 & 0.41 \\
\hline T4 & 0.49 \\
\hline T5 & 0.59 \\
\hline
\end{tabular}




\section{Results and discussions}

\subsection{Drag and lift coefficients}

Drag coefficient is a non-dimensional form of the drag force which can be determined as $C_{D}=F_{D} / \rho U|U|$ where $F_{D}$ is the drag force, $\rho$ is the fluid density and $U$ is the flow velocity. Fig. 3a shows the variation of drag coefficient $\left(C_{D}\right)$ with $R e$ for the smooth as well as for the marine growth wrapped cylinders. The values of $C_{D}$ depicted in Fig. 3a shows a slight rise on the drag coefficients as the Re Number increases. The $C_{D}$ coefficient for the smooth cylinder varied between 0.073 to 0.111 , while the $C_{D}$ values for the rough cylinder varied between $0.370-0.390$. This shows that marine growth increased the surface roughness, the total outer diameter and the added mass, which consequently resulted in comparatively higher drag coefficients. Further, the lift coefficient is an important parameter in VIV of circular cylinders and can be estimated as $C_{L}=F_{L} / \rho U|U|$, where $F_{L}$ is the transverse force. Fig. 3b shows the variation of $C_{L}$ with $\mathrm{Re}$. The range of $\mathrm{C}_{\mathrm{L}}$ for the smooth cylinder varied between 0.073-0.110 while the fouled cylinder has recorded lift coefficients ranging between $0.366-0.385$, which indicates that the surface roughness has significantly influenced the values of lift coefficients. The frequency of the lift force on a circular cylinder is consistent with the vortex shedding frequency, and the frequency of the drag force is double that of vortex shedding [9]. The range of drag and lift coefficients are in good agreement with the findings published earlier by [12-13].

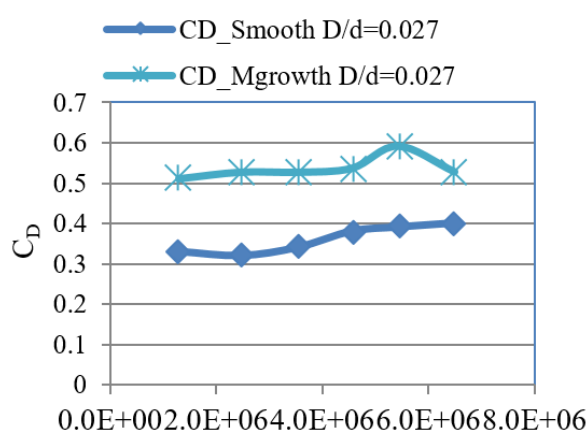

$\operatorname{Re}$

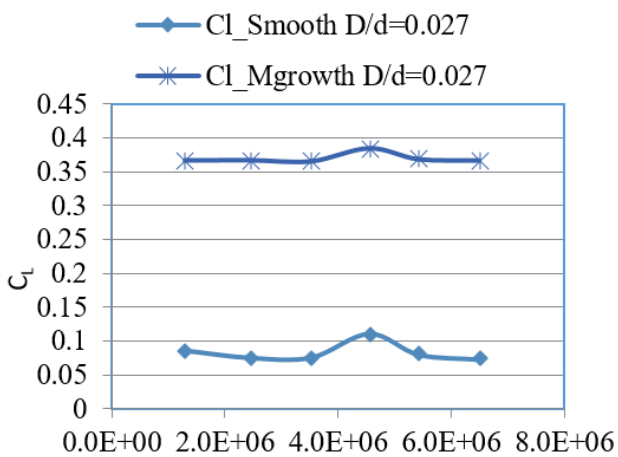

$\operatorname{Re}$

(a)

(b)

Fig. 3. Variation of drag (a) and lift coefficients with $R e$ Number for smooth and rough cylinders with $D / d=0.027$

\subsection{Response Amplitudes Ax/D and Ay/D}

Accurate estimation of the fatigue life of production riser experiencing vortex induced vibrations depends upon accurate estimation of the response amplitude and frequencies. However, accurate estimation of the response amplitudes and frequencies are in turn dependent upon several parameters such as surface roughness, hydrodynamic damping etc. Thus, in this section, the effect of marine growths on VIV response amplitude is discussed. The non-dimensional displacement amplitude of the cylinders along the flow directions $(A x / D)$ are depicted in Fig. 4a. The maximum response amplitude for the smooth and the marine growth wrapped cylinder have experienced the maximum response amplitude in the reduced velocity range between 4-7. The plot shows that the rough cylinder has experienced higher displacement although the ultimate response amplitude is in the range 
of 0.26D. Similarly, Fig. $4 \mathrm{~b}$ depicts the variation of cross-flow displacements of the cylinders $(A y / D)$. From the graph, one can observe that the response amplitude curve showed the initial, intermediate and upper branches which are typically observed for low mass-damping systems. Generally, the fouled cylinder has comparatively experienced higher responses amplitude.

\subsection{Response Amplitudes Ax/D and Ay/D}

Accurate estimation of the fatigue life of production riser experiencing vortex induced vibrations depends upon accurate estimation of the response amplitude and frequencies. However, accurate estimation of the response amplitudes and frequencies are in turn dependent upon several parameters such as surface roughness, hydrodynamic damping etc. Thus, in this section, the effect of marine growths on VIV response amplitude is discussed. The non-dimensional displacement amplitude of the cylinders along the flow directions $(A x / D)$ are depicted in Fig. 4a. The maximum response amplitude for the smooth and the marine growth wrapped cylinder have experienced the maximum response amplitude in the reduced velocity range between 4-7. The plot shows that the rough cylinder has experienced higher displacement although the ultimate response amplitude is in the range of $0.26 \mathrm{D}$. Similarly, Fig. $4 \mathrm{~b}$ depicts the variation of cross-flow displacements of the cylinders $(A y / D)$. From the graph, one can observe that the response amplitude curve showed the initial, intermediate and upper branches which are typically observed for low mass-damping systems. Generally, the fouled cylinder has comparatively experienced higher responses amplitude.

The maximum response in the cross-flow direction was recorded as $A y=1.6 \mathrm{D}$, observed in the reduced velocity interval ranging between $4-7$. The findings of this experimental study can be used to develop empirical models for prediction of drag and lift coefficients and responses for rough cylinders subjected to VIVs.

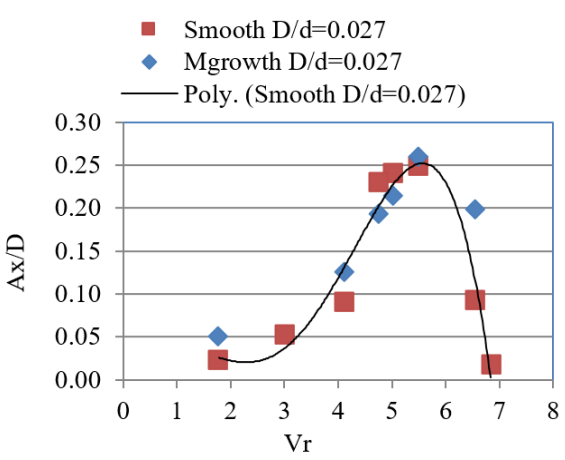

(a)

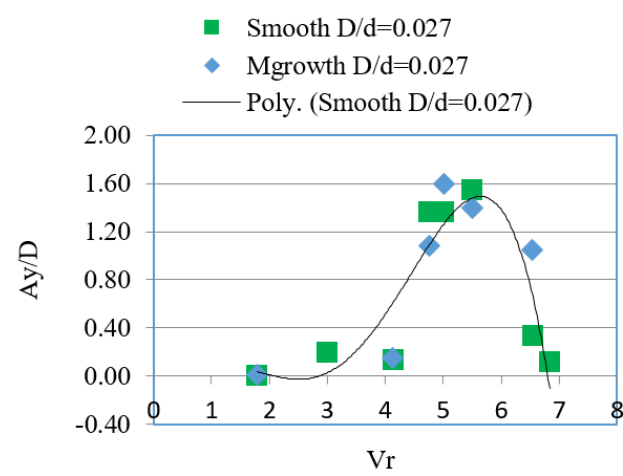

(b)

Fig. 4. Variation of drag (a) and lift coefficients with Re for smooth and rough cylinders with $D / d$ $=0.027$

\section{Conclusions}

The effect of marine growth on VIV forces and the corresponding response amplitudes have been determined and investigated experimentally in the wave tank using a circular cylinder with relative outer diameter $D / d=0.027 \mathrm{~mm}$. From the model tests, the following findings can be concluded: 
i) The $C_{D}$ coefficient for the smooth cylinder varied between $0.073-0.111$, while the drag coefficients for the rough cylinder varied between $0.370-0.390$.

ii) The range of $C_{L}$ for the smooth cylinder varied between 0.073-0.110 while the fouled cylinder has recorded lift coefficients ranging between 0.366-0.385, which indicates that the surface roughness has significantly influenced the values of lift coefficients.

iii) Generally, the fouled cylinder has comparatively experienced higher responses amplitude.

The authors would like to gratefully acknowledge their gratitude to Universiti Teknologi PETRONAS for support and encouragement.

\section{References}

[1] C. Feng, The measurement of vortex induced effects in flow past stationary and oscillating circular and D-section cylinders, Master Thesis, University of British Columbia, Vancouver, Canada (1968)

[2] P.W. Bearman, Vortex shedding from oscillating bluff bodies, Annual Review of Fluid Mechanics, 16(1),195-222, (1984)

[3] A. Khalak and C. Williamson, Motions, forces and mode transitions in vortex-induced vibrations at low mass-damping, J. of Fluids and Structures, 13(7), 813-851, (1999)

[4] A. Khalak and C. Williamson, Dynamics of a hydroelastic cylinder with very low mass and damping, J. of Fluids and Structures, 10(5), 455-472,(1996)

[5] C. Williamson and R. Govardhan, Vortex-induced vibrations, Annu. Rev. Fluid Mech., 36, 413-455, (2004)

[6] R.D Blevins and C.S. Coughran, Experimental investigation of vortex-induced vibration in one and two dimensions with variable mass, damping, and Reynolds number, J. of Fluids Engineering, 131(10), 1-7, (2009)

[7] A. Fage and J. Warsap, The effects of turbulence and surface roughness on the drag of a circular cylinder, Technical Report R\&M 1283, Aeronautical Research Council, London, United Kingdom (1930)

[8] F. Hover, H. Tvedt and M. Triantafyllou, Vortex-induced vibrations of a cylinder with tripping wires, J. of Fluid Mechanics, 448, 175-195, (2001)

[9] M. Zhao, K. Kaja, Y. Xiang and G. Yan, Vortex-induced vibration (VIV) of a circular cylinder in combined steady and oscillatory flow, Ocean Engineering, 73, 83-95, (2013)

[10] Y. Gao, D.S. Tan, B. Zhang and S.K. Tan, Experimental study on orbital response and flow behavior behind a freely suspended cylinder, Ocean Engineering, 108, 439-448. (2015)

[11]PETRONAS Technical Standards (PTS), Design of fixed offshore structures, PETRONAS, Kuala Lumpur, Malaysia (2014)

[12]B.M. Sumer and J. Fredsøe, Hydrodynamics around cylindrical structures, Advanced Series on Ocean Engineering, World Scientific, (2010)

[13] M. Zdravkovich, Flow around circular cylinders; Vol. 1. Fundamentals, J. of Fluid Mechanics, 350, 377-378, (2003) 